\title{
Toxic effects of 2,4-dichlorophenoxyacetic acid on human sperm function in vitro
}

\author{
Zhengyu Tan ${ }^{1}$, Jun Zhou1, Houyang Chen², Qianxing Zou 3 , Shiqi Weng ${ }^{3}$, Tao Luo ${ }^{3}$ \\ and Yuxin Tang ${ }^{1}$
}

\begin{abstract}
'Department of Urology, The Third Xiangya Hospital, The Central South University, Changsha, Hunan 410013, China ${ }^{2}$ Reproductive Medical Center, Jiangxi Provincial Maternal and Child Health Hospital, Nanchang, Jiangxi 330006, China ${ }^{3}$ Institute of Life Science, Nanchang University, Nanchang, Jiangxi 330031, China
\end{abstract}

(Received February 16, 2016; Accepted June 2, 2016)

\begin{abstract}
The herbicide 2,4-Dichlorophenoxyacetic acid (2,4-D) is globally used in agriculture and has been linked to human sperm abnormalities in vivo. However, its effects on ejaculated human spermatozoa in vitro have not been characterized. Therefore, we examined the effects of 2,4-D on the functions of ejaculated human spermatozoa in vitro, including: sperm motility, the ability to move through a viscous medium, capacitation, and the acrosome reaction. Different doses of 2,4-D (10 nM, $100 \mathrm{nM}$, $1 \mu \mathrm{M}, 10 \mu \mathrm{M}, 100 \mu \mathrm{M}$, and $200 \mu \mathrm{M})$ were applied to human spermatozoa prepared from normal fresh semen samples. The results indicated that 2,4-D did not affect the viability, capacitation, or spontaneous acrosome reactions of human spermatozoa, but it dose-dependently inhibited the total motility, progressive motility, ability to penetrate viscous medium, and progesterone-induced capacitation and acrosome reaction rates. These results suggest that exposure to 2,4-D and its accumulation in the seminal plasma and follicular fluid might increase the risk of infertility. Our findings provide new insights for understanding the male reproductive toxicity of 2,4-D.
\end{abstract}

Key words: 2,4-Dichlorophenoxyacetic acid, Acrosome reaction, Capacitation, Human spermatozoa, Motility

\section{INTRODUCTION}

2,4-Dichlorophenoxyacetic acid (2,4-D) is a chlorophenoxy herbicide that was developed in the 1940 s to selectively control broadleaf weeds. 2,4-D is the most used herbicide worldwide, and continues to be used in China. In the environment, it has a half-life of 2-14 days (Starrett, et al., 2000), and it can persist in agricultural soils for longer periods. It can be absorbed by the human body after long periods of contact, but it is cleared quickly via urine without being metabolized or accumulating in tissues (Sauerhoff et al., 1977). Despite this, 2,4-D has been linked to human genotoxicity (Charles et al., 2001), carcinogenesis (Glickman et al., 2004), neurotoxicity (Bongiovanni et al., 2007), and reproductive toxicity (Arbuckle et al., 1999a). In addition, 2,4-D can cause abnormal embryogenesis (Weselak et al., 2007). Some studies have reported that it can also be detected in human spermatozoa (Swan et al., 2003) and has adverse effects on human semen. Arbuckle et al. (1999b) studied the semen quality of 32 male farmers in Ontario who were exposed to 2,4-D in their daily work, and found cases of oligoasthenozoospermia, asthenozoospermia, and teratozoospermia among them. About $50 \%$ of the semen samples had a 2,4-D level at or above the detection limit of $20 \mathrm{nM}$ and the highest level of 2,4-D following longterm contact was $2.94 \mu \mathrm{M}$. Lerda and Rizzi (1991) found a similar relationship. These studies strongly suggest that 2,4-D interferes with human sperm production. However, the mechanism has not been defined, and there has been no research on whether there are direct effects on spermatozoa in vitro.

Spermatozoa are produced in the testis and mature in the epididymis. After being ejaculated into the female reproductive tract, there is a delay in the ability of sperm to fertilize, known as capacitation. To penetrate the egg vestments, enzymes in the acrosome have to be released in a process called the acrosome reaction. Any distur-

Correspondence: Yuxin Tang (E-mail: mmcct@126.com; tp123kk@163.com) 
bance in any of these processes will impair sperm function. In this study, we assessed the effects of 2,4-D on sperm motility, viability, hyperactivation, capacitation, and the acrosome reaction in vitro.

\section{MATERIALS AND METHODS}

\section{Sample collection}

Semen samples were collected by masturbation from healthy 25-35-year-old donors with a documented reproductive history over the previous 2 years and normal semen parameters (sperm count $>40$ million $/ \mathrm{mL}$, total motility $>60 \%$ and progressive motility $>45 \%$ ). They had different careers and backgrounds, and lived in Changsha (Hunan, China). Collection of the samples and the research protocol were approved by the Research Center of Male Disease of the Third Xiangya Hospital. Spermatozoa were purified by direct swim-up in human tubal fluid medium (HTF, Millipore, Bedford, MA, USA) as described previously (Alasmari et al., 2013) and were adjusted to a final concentration of 80 million cells $/ \mathrm{mL}$. Tubal fluid medium is a capacitating medium containing $25 \mathrm{mM} \mathrm{NaHCO}_{3}$ and 3\% human serum albumin, which mimics the physiological environment in the female reproductive tract as described previously (Luo et al., 2015). According to Arbuckle et al. the concentration of 2,4-D in human semen ranges from being undetectable to $2.94 \mu \mathrm{M}$. Therefore, in this study, $50 \mu \mathrm{L}$ aliquots of sperm suspensions from each single donor were mixed with equal volumes of HTF medium containing different concentrations of 2,4-D to final doses of $10 \mathrm{nM}, 100 \mathrm{nM}$, $1 \mu \mathrm{M}, 10 \mu \mathrm{M}, 100 \mu \mathrm{M}$, and $200 \mu \mathrm{M}$. All samples were incubated in $5 \% \mathrm{CO}_{2}$ in humid air at $37^{\circ} \mathrm{C}$. Sperm samples from 7-10 donors were examined in each experiment. The 2,4-D was purchased from Sigma-Aldrich Corporation (St. Louis, MI, USA) and the 2,4-D stock solution was prepared using dimethyl sulfoxide at the concentration of $100 \mathrm{mM}$ and stored at $4^{\circ} \mathrm{C}$.

\section{Assessment of sperm motility and viability}

Sperm motility was evaluated using a computer-assisted sperm analysis (CASA) system (WLJY-9000, WeiLi Co., Ltd., Beijing, China) after $1 \mathrm{hr}$ of incubation, and progressive motility and total motility percentages were measured for 200 spermatozoa. Progressive motility and fertilization competence are closely related, because spermatozoa exhibiting progressive motility can easily penetrate the mucus in the female reproductive tract. Total motility is defined as the sum of progressive motility (PR) and the non-progressive motility (NP). According to the fifth edition of WHO laboratory manual for the examination and processing of human semen, the limited value of normal progressive motility is $32 \%$ and the total motility is $40 \%$. After $4 \mathrm{hr}$ of incubation with different concentrations of 2,4-D, sperm viability was tested with an eosin staining kit (Nanchang Royo Biotech Co. Ltd., Nanchang, China). The heads of dead spermatozoa were stained red with eosin and living cells were not stained. Two hundred spermatozoa were counted for each sample.

\section{Penetration of artificial viscous medium}

In the penetration assay, $1 \%$ methylcellulose solution was used to mimic the viscous conditions in the female reproductive tract (Alasmari et al., 2013). Methylcellulose was dissolved in HTF medium and introduced into $7.5-\mathrm{cm}$ long flattened capillary tubes $(1.0-\mathrm{mm}$ inner depth; Elite Medical Co. Ltd., Nanjing, China) with one end sealed using plasticine. After $2 \mathrm{hr}$ of incubation (in $5 \% \mathrm{CO}_{2}$ in humid air at $37^{\circ} \mathrm{C}$ ), sperm suspensions were mixed with different concentrations of 2,4-D and incubated for $1 \mathrm{hr}$ at $37^{\circ} \mathrm{C}$ under $5 \% \mathrm{CO}_{2}$ in humid air. Next, the open side of the capillary tubes was inserted into a sperm suspension and incubated for $1 \mathrm{hr}$. Then, the tubes were removed and examined with a Leica DM2500 phase contrast microscope (Leica, Wetzlar, Germany). Spermatozoa were counted at 1 and $2 \mathrm{~cm}$ from the open end of the capillary and the mean sperm count per field (at $10 \times$ magnification) was calculated. Values of parallel, untreated samples were used as controls. Another series of similar experiments were designed to evaluate the effect of progesterone (P4) stimulation and suspensions without $\mathrm{P} 4$ or 2,4-D were used as controls.

\section{Evaluation of capacitation and the acrosome reaction}

To evaluate whether 2,4-D influences capacitation and the acrosome reaction of spermatozoa in vitro (De Jonge, 2005), four sets of experiments were designed. For set 1 , we used a control sperm suspension and three samples with different concentrations of 2,4-D (10 nM, $1 \mu \mathrm{M}, 100 \mu \mathrm{M})$ in HTF for $4 \mathrm{hr}$, together with an uncapacitated control sample, which was incubated in HS medium (an uncapacitated medium containing $135 \mathrm{mM} \mathrm{NaCl}$, $5 \mathrm{mM} \mathrm{KCl}, 1 \mathrm{mM}, \mathrm{MgSO}_{4}, 2 \mathrm{mM} \mathrm{CaCl}, 20 \mathrm{mM}$ HEPES, $5 \mathrm{mM}$ glucose, $10 \mathrm{mM}$ lactic acid, and $1 \mathrm{mM} \mathrm{Na-pyru-}$ vate adjusted to $\mathrm{pH} 7.4$ with $\mathrm{NaOH}$ ), to assess the influence of 2,4-D on capacitation. In set 2 , sperm suspensions were first capacitated in HTF for $4 \mathrm{hr}$ and different concentrations of 2,4-D and DMSO were added for an additional hour to assess the influence of 2,4-D on the acrosome reaction. In set 3 , all suspensions were prepared as described in set 1 with the addition of $20 \mu \mathrm{M}$ progesterone 
2,4-D compromises human sperm function

(P4) to evaluate the influence of 2,4-D on progesteroneinduced capacitation. For set 4 , samples were prepared as in set 2 with $20 \mu \mathrm{M}$ P 4 added to each group after $4 \mathrm{hr}$ incubation to assess the influence of 2,4-D on the P4-induced acrosome reaction. Chlortetracycline (CTC) staining was used to evaluate capacitation and the acrosome reaction of spermatozoa (Perry et al., 1995). The stained spermatozoa were examined using a Leica DM2500 fluorescence microscope using an $\mathrm{Hg}$ lamp passed through a 340-380 nm filter and fluorescence emission was assessed via a DM 400 dichromatic mirror (Leica "A" filter). There were three different CTC staining patterns: "F" represents the characteristics of uncapacitated spermatozoa and looks uniformly bright; "B" represents capacitated but acrosome-intact sperm, in which a dark stripe can be seen at the equatorial zone; and "AR" represents spermatozoa that had undergone acrosomal exocytosis so that the acrosome had disappeared. The sum of "B" and "AR" patterns was taken as the number of capacitated spermatozoa. In all, 200 spermatozoa were counted for each experimental sperm suspension.

\section{Statistical analyses}

All data are reported as the mean \pm standard error of the mean (S.E.M.). Differences between the control and experimental groups were compared using One-way ANOVA analysis. Statistically significant differences were determined at $P<0.05$ using GraphPad Prism statistical software (version 5.01; http://www.graphpad/com/ prism).

\section{RESULTS}

\section{2,4-D has an adverse effect on sperm motility but no effect on viability}

In all of our experiments, 2,4-D had no effect on sperm viability as determined by vital staining (Fig. 1). However, Both progressive motility and total motility as shown by CASA were significantly inhibited by 2,4-D at $200 \mu \mathrm{M}, 100 \mu \mathrm{M}$ and $10 \mu \mathrm{M}$ (Fig. 2). Both progressive and total motility exhibited similar decreases because of the function of 2,4-D concentration. At 2,4-D concentrations of $100 \mu \mathrm{M}$ and $200 \mu \mathrm{M}$, both progressive and total motility were lower than the limited value of normal.

\section{2,4-D significantly decreased the ability of human spermatozoa to penetrate viscous medium}

In the experiments without $\mathrm{P} 4$ treatment, we found that 2,4-D inhibited sperm penetration into a methylcellulose medium in a dose-dependent manner. Sperm in suspen- sions treated with P4 exhibited significantly improved penetration compared with groups without P4 treatment. When induced by $\mathrm{P} 4$, the toxic effect of 2,4-D on penetration is likely to become more pronounced. Doses of 2,4-D at or above $1 \mu \mathrm{M}(1 \mu \mathrm{M}, 10 \mu \mathrm{M}, 100 \mu \mathrm{M}, 200 \mu \mathrm{M})$ showed a significant tendency to decrease sperm motility compared with the controls (Fig. 3). This suggests that 2,4-D adversely affects the ability of spermatozoa to pass through viscous fluids in the female reproductive tract. Because hyperactivated motility of capacitated spermatozoa is important for traversing the reproductive tract, we suspect that elevated levels of 2,4-D influence this aspect of sperm function.

\section{2,4-D treatment affects progesterone-induced capacitation and the acrosome reaction}

We found that 2,4-D treatment did not affect the capacitation or acrosome reaction rates of sperm not treated with P4. However, sperm suspensions treated with P4 showed decreased competence for capacitation and the acrosome reaction when treated with 2,4-D. The observed decrease was dose-dependent (Fig. 4). These results suggest that 2,4-D impairs sperm functions stimulated by $\mathrm{P} 4$ in the female reproductive tract. However, the mechanism of this impairment requires further exploration.

\section{DISCUSSION}

2,4-D has always been considered relatively safe for humans. However, several toxicological studies

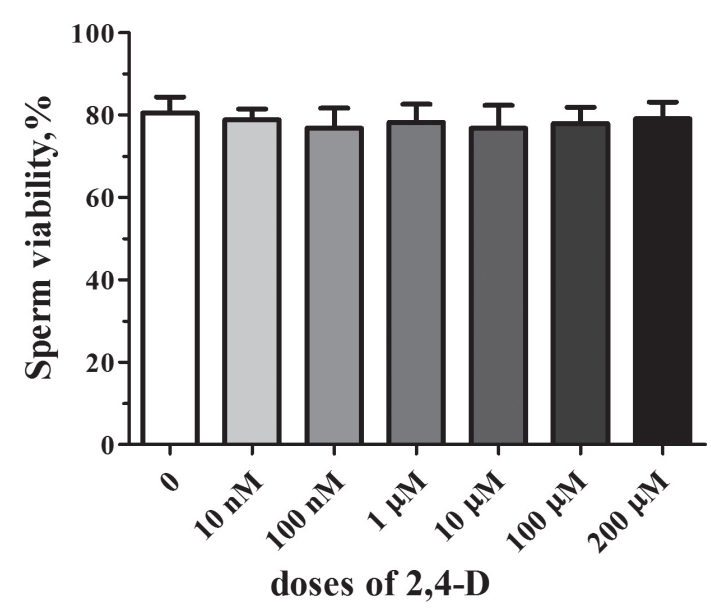

Fig. 1. The effect of 2,4-D on sperm viability in vitro. Ejaculated human sperm were incubated with different doses of 2,4-D in HTF medium at $37^{\circ} \mathrm{C}$ and $5 \% \mathrm{CO}_{2}$ for $4 \mathrm{hr}$, A minimum of 200 sperm were counted for each assay. Bar: mean \pm S.E.M. *-p $<0.05, * *$-p $<0.001$ and $* * *-\mathrm{p}<0.0001, \mathrm{n}=10$. 


\section{Z. Tan et al.}
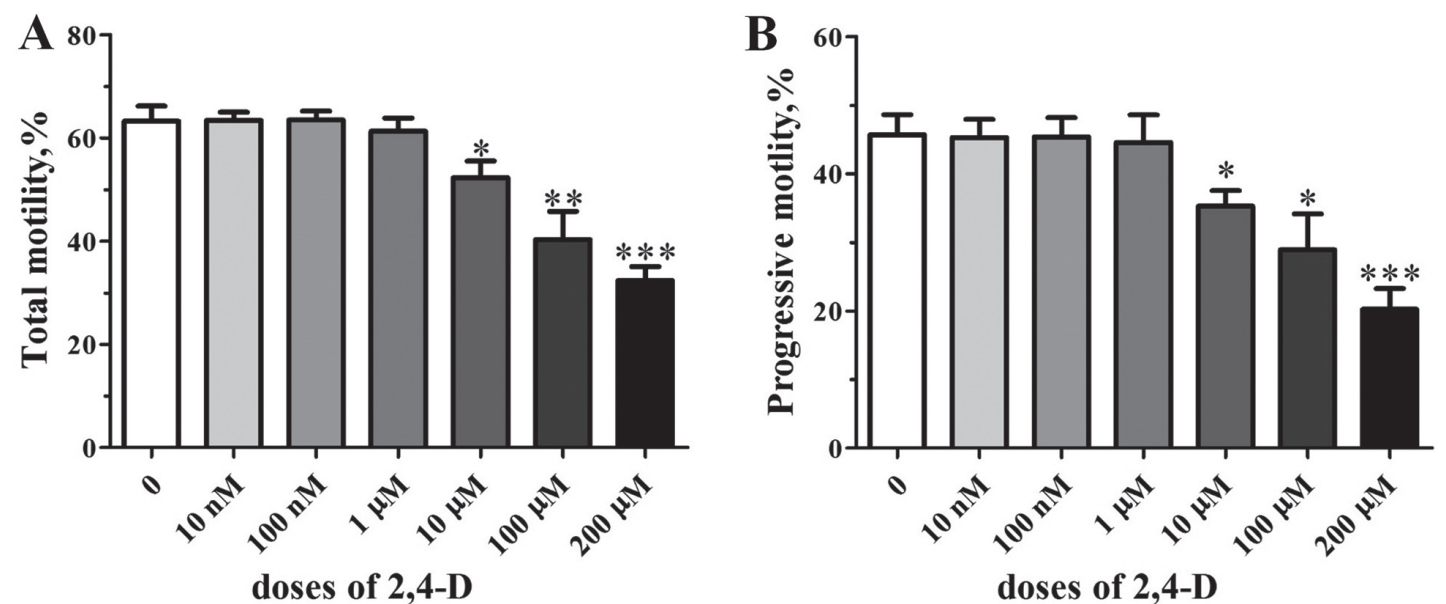

Fig. 2. The effect of 2,4-D on sperm motility and viability in vitro. Ejaculated human sperm were incubated with different doses of 2,4-D at $37^{\circ} \mathrm{C}$ and $5 \% \mathrm{CO}_{2}$ for $1 \mathrm{hr}$. Motility, including progressive motility (A) and total motility (B) were analyzed by Computer-Assisted Sperm Analysis (CASA). A minimum of 200 sperm were counted for each assay. Bar: mean \pm S.E.M. $*_{-} \mathrm{p}<0.05, * *-\mathrm{p}<0.001$ and ${ }^{* * *}$ - $\mathrm{p}<0.0001, \mathrm{n}=10$.
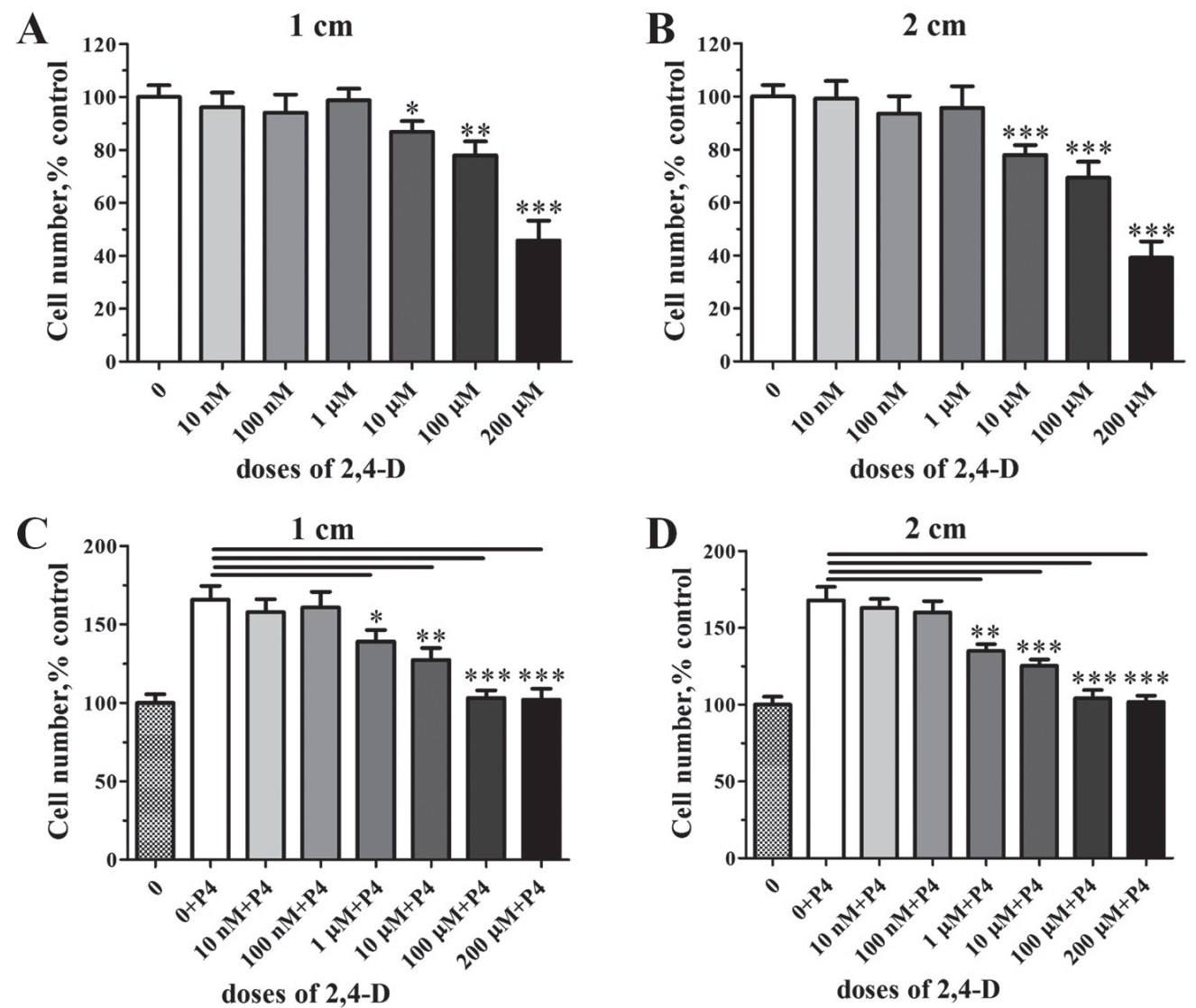

Fig. 3. The effect of 2,4-D on human sperm ability to penetrate into viscous medium. Human sperm were exposed to different concentrations of 2,4-D (with or without progesterone). Cell density (percentage of control) of $1 \mathrm{~cm}$ and $2 \mathrm{~cm}$ into methylcellulose was shown. Bar: mean \pm S.E.M. ${ }^{*} \mathrm{p}<0.05,{ }^{* *} \mathrm{p}<0.001$ and ${ }^{* * *} \mathrm{p}<0.0001, \mathrm{n}(3 \mathrm{a}, 3 \mathrm{~b})=12, \mathrm{n}(3 \mathrm{c}, 3 \mathrm{~d})=9$. 

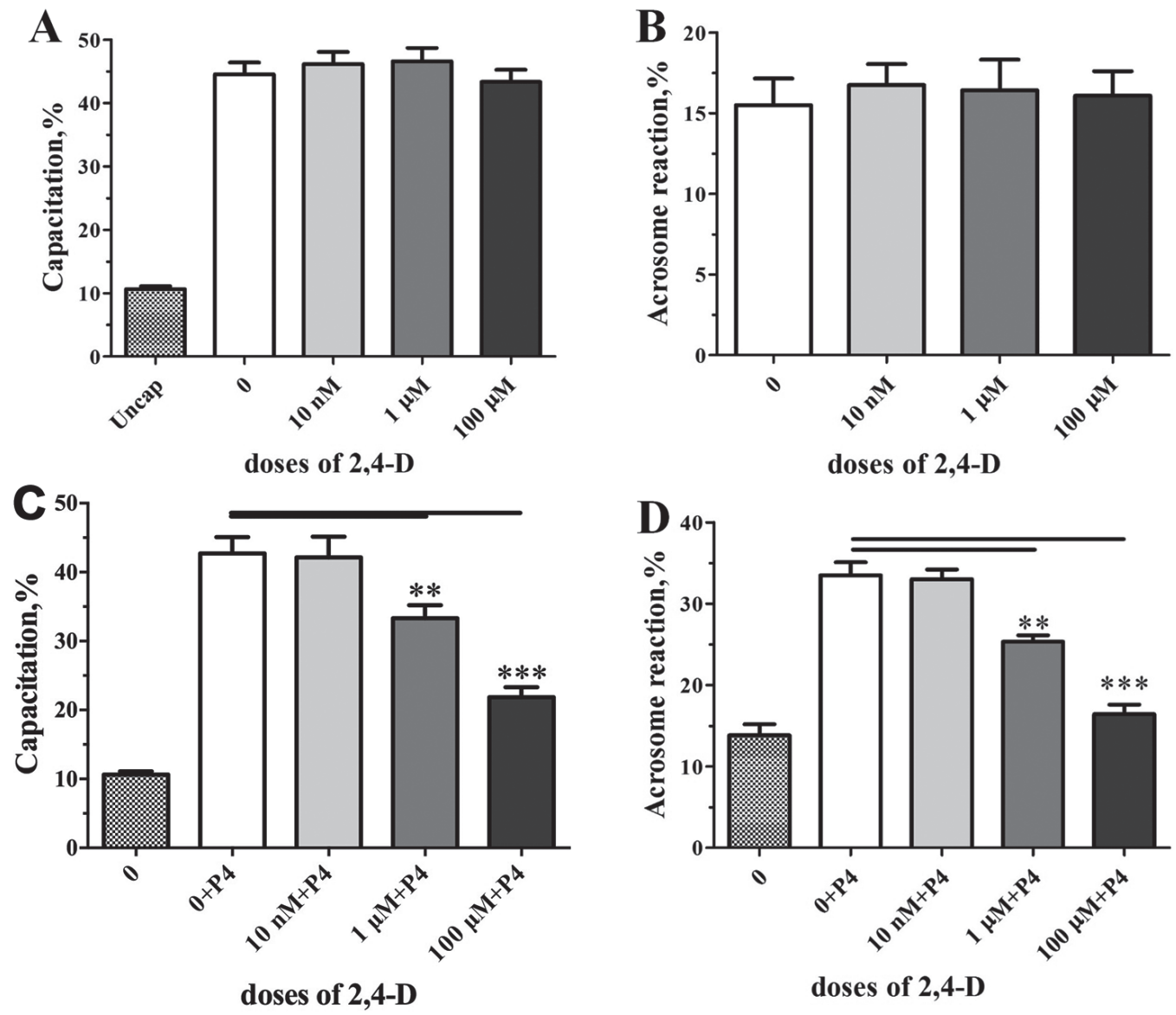

Fig. 4. Assessment of the acrosomal status of human sperm exposed to 2,4-D in vitro. The human sperm were incubated in capacitating HTF medium containing $0-200 \mu \mathrm{M} 2,4-\mathrm{D}$ (with or without progesterone) at $37^{\circ} \mathrm{C}$ in a $5 \% \mathrm{CO}_{2}$ for $1 \mathrm{hr}$. The ratios of capacitation were assessed by chlortetracycline (CTC) staining. The acrosome reactions were evaluated by CTC staining. Bar: mean \pm S.E.M., ${ }^{*}$-p $<0.05, * *$ - $p<0.001$ and ${ }^{* * *}$ - $\mathrm{p}<0.0001, \mathrm{n}=11$.

have reported that long-term contact with 2,4-D might be toxic for mammals. Arbuckle et al. and Lerda et al. demonstrated the toxicity of 2,4-D on mammalian spermatozoa. In contrast, other studies found no evidence of 2,4-D contact causing significant adverse effects on reproduction (Marty et al., 2013). Therefore, the actual influence of 2,4-D on human spermatozoa is uncertain, and in vitro experiments might demonstrate its influence from another perspective.

Here we used different concentrations of 2,4-D $(10 \mathrm{nM}, 100 \mathrm{nM}, 1 \mu \mathrm{M}, 10 \mu \mathrm{M}, 100 \mu \mathrm{M}, 200 \mu \mathrm{M})$ study the in vitro effects of 2,4-D on human sperm functions. High doses of 2,4-D (10 $\mu \mathrm{M}, 100 \mu \mathrm{M}, 200 \mu \mathrm{M})$ significantly inhibited human sperm motility. It is noteworthy that 2,4-D did not damage sperm viability after shortterm contact. Instead, our studies on the ability of treated sperm to penetrate viscous medium, induce capacitation, and undergo the P4-induced acrosome reaction suggested that 2,4-D might hamper these physiological processes. They also suggest a relationship between the functions of 2,4-D and P4. However, according to Arbuckle et al., the highest level of 2,4-D in the semen of men experiencing long-term exposure was $2.94 \mu \mathrm{M}$. The doses $(10-200 \mu \mathrm{M})$ of 2,4-D that inhibit sperm function in vitro are far above those found in human semen under common exposure conditions (Thomas et al., 2010).

Our experiments provide multiple important observations. First, spermatozoa are not killed when in brief contact with 2,4-D, even at a high concentration $(200 \mu \mathrm{M})$. Second, at concentrations below $10 \mu \mathrm{M}, 2,4-\mathrm{D}$ alone has little influence on human sperm. However, in the presence of P4, we found that doses of 2,4-D as low as $1 \mu \mathrm{M}$ inhibited P4-induced capacitation and acrosome reaction rates. The viscous medium penetration assay can com- 
prehensively evaluate the motility of spermatozoa. Compared with the results obtained from the previous observations of progressive motility total motility, the results of the penetration assay without P4 indicated a similar trend. This suggests that 2,4-D could inhibit the function of spermatozoa immediately following ejaculation into the reproductive tract, prior to being affected by $\mathrm{P} 4$. However, when the spermatozoa approach the ovum, the spermatozoa will be affected by hormone (Fraser, 1998). In vitro experiments with $\mathrm{P} 4$-induced spermatozoa better evaluate the physiological processes taking place prior to fertilization. According to our results, low concentrations of 2,4-D $(>1 \mu \mathrm{M})$ decreased the function of P4-induced spermatozoa, which indicates that toxicity of 2,4-D could be much more significant in the female reproductive tract. However, even though our studies showed that 2,4-D impaired human sperm functions, the mechanism is yet unknown.

Previous reports provide evidence in support of multiple hypotheses regarding the mechanism of 2,4-D toxicity (Stebbins-Boaz et al., 2004; Lin and Garry, 2000). One hypothesis is that 2,4-D influences the endocrine system or acts directly to disturb the growth of the reproductive system. Our research has shown that 2,4-D can affect mature spermatozoa directly in vitro. Our experiments on sperm penetration of viscous medium and capacitation and acrosome reaction suggest that the effect of 2,4-D might have a close relationship with the action of $\mathrm{P} 4$, which can activate the CatSper channel on the membrane of sperm to induce $\mathrm{Ca}^{2+}$ influx (Strünker et al., 2011). Many studies have found that intracellular $\mathrm{Ca}^{2+}$ concentration plays an essential role in the modulation of a range of activities that are pivotal to sperm function (Lin and Garry, 2000). Therefore, although there has been little research about the link between 2,4-D and calcium channels, intracellular $\mathrm{Ca}^{2+}$ might be a factor through which 2,4-D could influence human sperm function. If the $\mathrm{Ca}^{2+}$ in human spermatozoa is decreased by high doses of 2,4-D, its effect on sperm motility could be explained.

Additionally, previous studies have demonstrated that 2,4-D induces a high level of oxidative stress as determined by an analysis of antioxidant enzymes, levels of glutathione, and lipid peroxidation (Bukowska, 2003). Oxidative stress will lead to cell death, and this can be prevented by drugs such as Quercetin (Mi et al., 2007). Even though we found no significant difference in sperm viability between the control and experimental groups, oxidative stress can still be considered worthy of further study.

In mature mammalian spermatozoa, post-translational protein modifications serve as important regulators of sperm function. Phosphorylation of tyrosine residues is essential for the maintenance of sperm motility, capacitation and the acrosome reaction (Naz and Rajesh, 2004). That suggests that we should assess the level of tyrosine phosphorylation in spermatozoa incubated with 2,4-D.

In conclusion, we examined the effects of 2,4-D on human sperm function in vitro, and two possible mechanisms have been discussed. Research on the possible mechanism of this pesticide's reproductive toxicity to human spermatozoa will provide a more profound understanding of the biological impact of 2,4-D and enable the development of preventive measures and treatments.

\section{ACKNOWLEDGMENTS}

This work was supported by the the New Xaingya Talent Project of the Third xiangya hospital of Central South University, the project number is "20160306".

Conflict of interest---- The authors declare that there is no conflict of interest.

\section{REFERENCES}

Alasmari, W., Costello, S., Correia, J., Oxenham, S.K., Morris, J., Fernandes, L., Ramalho-Santos, J., Kirkman-Brown, J., Michelangeli, F., Publicover, S. and Barratt, C.L. (2013): $\mathrm{Ca} 2+$ signals generated by CatSper and $\mathrm{Ca} 2+$ stores regulate different behaviors in human sperm. J. Biol. Chem., 288, 62486258.

Arbuckle, T.E., Savitz, D.A., Mery, L.S. and Curtis, K.M. (1999a): Exposure to phenoxy herbicides and the risk of spontaneous abortion. Epidemiology, 10, 752-760.

Arbuckle, T.E., Schrader, S.M., Cole, D., Hall, J.C., Bancej, C.M., Turner, L.A. and Claman, P. (1999b): 2,4-Dichlorophenoxyacetic acid residues in semen of Ontario farmers. Reprod. Toxicol., 13, 421-429.

Bongiovanni, B., De Lorenzi, P., Ferri, A., Konjuh, C., Rassetto, M., Evangelista de Duffard, A.M., Cardinali, D.P. and Duffard, R. (2007): Melatonin decreases the oxidative stress produced by 2,4-dichlorophenoxyacetic acid in rat cerebellar granule cells. Neurotox. Res., 11, 93-99.

Bukowska, B. (2003): Effects of 2,4-D and its metabolite 2,4dichlorophenol on antioxidant enzymes and level of glutathione in human erythrocytes. Comp. Biochem. Physiol. C Toxicol. Pharmacol., 135, 435-441.

Charles, J.M., Hanley, T.R.Jr., Wilson, R.D., van Ravenzwaay, B. and Bus, J.S. (2001): Developmental toxicity studies in rats and rabbits on 2,4-dichlorophenoxyacetic acid and its forms. Toxicol. Sci., 60, 121-131.

De Jonge, C. (2005): Biological basis for human capacitation. Hum. Reprod. Update, 11, 205-214.

Fraser, L.R. (1998): Sperm capacitation and the acrosome reaction. Hum. Reprod., 13 Suppl. 1, 9-19.

Glickman, L.T., Raghavan, M., Knapp, D.W., Bonney, P.L. and Dawson, M.H. (2004): Herbicide exposure and the risk of transitional cell carcinoma of the urinary bladder in Scottish Terriers. 
2,4-D compromises human sperm function

J. Am. Vet. Med. Assoc., 224, 1290-1297.

Lerda, D. and Rizzi, R. (1991): Study of reproductive function in persons occupationally exposed to 2,4-dichlorophenoxyacetic acid (2,4-D). Mutat. Res., 262, 47-50.

Lin, N. and Garry, V.F. (2000): In vitro studies of cellular and molecular developmental toxicity of adjuvants herbicides, and fungicides commonly used in Red River Valley, Minnesota. J. Toxicol. Environ. Health A, 60, 423-439.

Luo, T., Li, N., He, Y.Q., Weng, S.Q., Wang, T., Zou, Q.X. and Zeng, X.H. (2015): Emodin inhibits human sperm functions by reducing sperm $[\mathrm{Ca}(2+)] \mathrm{i}$ and tyrosine phosphorylation. Reprod. Toxicol., 51, 14-21.

Marty, M.S., Neal, B.H., Zablotny, C.L., Yano, B.L., Andrus, A.K., Woolhiser, M.R., Boverhof, D.R., Saghir, S.A., Perala, A.W., Passage, J.K., Lawson, M.A., Bus, J.S., Lamb, J.C.4th. and Hammond, L. (2013): An F1-extended one-generation reproductive toxicity study in $\mathrm{Crl}: \mathrm{CD}(\mathrm{SD})$ rats with 2,4-dichlorophenoxyacetic acid. Toxicol. Sci., 136, 527-547.

Mi, Y.L., Zhang, C.Q. and Taya, K. (2007): Quercetin protects spermatogonial cells from 2,4-D-induced oxidative damage in embryonic chickens. J. Reprod. Dev., 53, 749-754.

Naz, R.K. and Rajesh, P.B. (2004): Role of tyrosine phosphorylation in sperm capacitation / acrosome reaction. Reprod. Biol. Endocrinol., 2, 75 .

Perry, R.L., Naeeni, M., Barratt, C.L., Warren, M.A. and Cooke, I.D. (1995): A time course study of capacitation and the acrosome reaction in human spermatozoa using a revised chlortetracycline pattern classification. Fertil Steril., 64, 150-159.

Sauerhoff, M.W., Braun, W.H., Blau, G.E. and Gehring, P.J. (1977):
The fate of 2,4-dichlorophenoxyacetic acid (2,4-D) following oral administration to man. Toxicology, 8, 3-11.

Starrett, S.K., Christians, N.E. and Al Austin, T. (2000): Movement of herbicides under two irrigation regimes applied to turfgrass. Adv. Environ. Res., 4, 169-176.

Stebbins-Boaz, B., Fortner, K., Frazier, J., Piluso, S., Pullen, S., Rasar, M., Reid, W., Sinclair, K. and Winger, E. (2004): Oocyte maturation in Xenopus laevis is blocked by the hormonal herbicide, 2,4-dichlorophenoxy acetic acid. Mol. Reprod. Dev., 67, 233-242.

Strünker, T., Goodwin, N., Brenker, C., Kashikar, N.D., Weyand, I., Seifert, R. and Kaupp, U.B. (2011): The CatSper channel mediates progesterone-induced $\mathrm{Ca} 2+$ influx in human sperm. Nature, 471, 382-386.

Swan, S.H., Kruse, R.L., Liu, F., Barr, D.B., Drobnis, E.Z., Redmon, J.B., Wang, C., Brazil, C., Overstreet, J.W. and Study for Future Families Research Group (2003): Semen quality in relation to biomarkers of pesticide exposure. Environ. Health Perspect., 111, 1478-1484.

Thomas, K.W., Dosemeci, M., Hoppin, J.A., Sheldon, L.S., Croghan, C.W., Gordon, S.M., Jones, M.L., Reynolds, S.J., Raymer, J.H., Akland, G.G., Lynch, C.F., Knott, C.E., Sandler, D.P., Blair, A.E. and Alavanja, M.C. (2010): Urinary biomarker, dermal, and air measurement results for 2,4-D and chlorpyrifos farm applicators in the Agricultural Health Study. J. Expo. Sci. Environ. Epidemiol., 20, 119-134.

Weselak, M., Arbuckle, T.E., Wigle, D.T. and Krewski, D. (2007): In utero pesticide exposure and childhood morbidity. Environ. Res., 103, 79-86. 